\title{
Mapping regulatory variants controlling gene expression in drought response and tolerance in maize
}

Shengxue Liu ${ }^{1 \dagger}$, Cuiping $\mathrm{Li}^{2 \dagger}$, Hongwei Wang ${ }^{3}$, Shuhui Wang ${ }^{1}$, Shiping Yang ${ }^{1}$, Xiaohu Liu', Jianbing Yan ${ }^{4}$, Bailin Li ${ }^{5}$, Mary Beatty ${ }^{5}$, Gina Zastrow-Hayes ${ }^{5}$, Shuhui Song ${ }^{2^{*}}$ and Feng Qin ${ }^{1 *}$ (i)

* Correspondence: songshh@big.ac. cn; qinfeng@cau.edu.cn

†Shengxue Liu and Cuiping Li contributed equally to this work. ${ }^{1}$ State Key Laboratory of Plant Physiology and Biochemistry, College of Biological Sciences, China Agricultural University, Beijing 100193, China

${ }^{2}$ National Genomics Data Center \& CAS Key Laboratory of Genome Sciences and Information, Beijing Institute of Genomics, Chinese Academy of Sciences, and China National Center for Bioinformation, Beijing 100101, China Full list of author information is available at the end of the article

\begin{abstract}
Background: Gene expression is a key determinant of cellular response. Natural variation in gene expression bridges genetic variation to phenotypic alteration. Identification of the regulatory variants controlling the gene expression in response to drought, a major environmental threat of crop production worldwide, is of great value for drought-tolerant gene identification.

Results: A total of 627 RNA-seq analyses are performed for 224 maize accessions which represent a wide genetic diversity under three water regimes; 73,573 eQTLs are detected for about 30,000 expressing genes with high-density genome-wide single nucleotide polymorphisms, reflecting a comprehensive and dynamic genetic architecture of gene expression in response to drought. The regulatory variants controlling the gene expression constitutively or drought-dynamically are unraveled. Focusing on dynamic regulatory variants resolved to genes encoding transcription factors, a drought-responsive network reflecting a hierarchy of transcription factors and their target genes is built. Moreover, 97 genes are prioritized to associate with drought tolerance due to their expression variations through the Mendelian randomization analysis. One of the candidate genes, Abscisic acid 8'-hydroxylase, is verified to play a negative role in plant drought tolerance.
\end{abstract}

Conclusions: This study unravels the effects of genetic variants on gene expression dynamics in drought response which allows us to better understand the role of distal and proximal genetic effects on gene expression and phenotypic plasticity. The prioritized drought-associated genes may serve as direct targets for functional investigation or allelic mining.

Keywords: Regulatory variants, Gene expression, Drought response, Stress tolerance, Maize 


\section{Background}

Maize is an important crop for food, forage, and industrial compounds [1, 2] but also serves as a model organism for its abundant genetic polymorphisms and quick linkage decay on a genome-wide scale [3]. Drought stress is a major environmental factor which limits crop production worldwide [4]; thus, understanding the molecular mechanisms and identifying the functional variants underlying drought tolerance are critical for the trait improvement. However, as a complex quantitative trait, genetic dissection for plant drought tolerance is difficult. Although genome-wide association studies (GWAS) facilitate the detection of genetic and phenotypic associations, identification of the causal genes or DNA variants that mechanistically affect the trait is still challenging $[5,6]$. Cumulative evidence suggests that genetic variants influence complex traits through modulating gene expression; highlighting the functional importance in regulatory variants. For instance, a short interspersed nuclear element (SINE), AluJb, located $20 \mathrm{~kb}$ upstream of $\operatorname{lin}-28$ homolog B (LIN28B) drives the majority of LIN28B expression in a substantial number of human tumors for oncogene activation [7]. Teosinte branched $1(\mathrm{~Tb} 1)$ gene expression is activated due to a transposon insertion $60 \mathrm{~kb}$ upstream of $T b 1$, which contributes to maize domestication from its wild ancestor teosinte (Zea mays ssp. parviglumis) [8]. A genetic insertion upstream of in Vegetative to generative transition 1 ( $v g t 1$ ) represses gene expression and promotes maize flowering time [9]. Genetic variations in ZmVPP1 and ZmNAC111I gene regulatory regions affect gene expression which are associated with the natural variation in drought tolerance [6, 10]. Recently, a 2-bp deletion, $9.5 \mathrm{~kb}$ upstream of ZmRAVL1, was identified as the causal variant responsible for the enlarged leaf angles in maize [11].

The genetic basis of gene expression variation, also known as quantitative trait loci (eQTLs), was studied in many model organisms [12-14]. However, a classical strategy to identify the genetic determinants for gene expression through analyzing a population of recombinant offspring of two parent strains usually detects large genomic intervals lacking accurate information for causal gene prediction. Recently, association analysis, based on genetic linkage disequilibrium (LD) at a natural population level, exploits historical and evolutionary recombination with high-density markers, providing an alternative strategy for eQTL dissection $[15,16]$. Due to the quick linkage disequilibrium (LD) decay in the maize genome, resolution of the detected regions in association studies often reaches a single-gene level $[6,17]$. Moreover, given that gene expression is spatio-temporal dependent, eQTL architecture can change dynamically during development or in response to environmental stimuli. Such dynamics are considered to play an important functional role and have been thoroughly explored in multiple types of human tissues [18], but rarely in other species.

More importantly, gene expression alterations are regarded as important molecular phenotypes that link genotypes to molecular functions and plant fitness, whose regulation is of fundamental importance to stress response and adaptation [19]. Strategies, such as Mendelian randomization (MR) analysis and transcriptome-wide association study (TWAS), were recently developed to identify expression-trait associations, which prioritize candidate genes associated with the trait due to gene expression variations $[20,21]$. Thus, a comprehensive and accurate understanding of the plasticity of gene expression architecture in response to stress is essential but demanding. 
In this research, 627 RNA-seq data of 224 maize accessions (inbred lines) subjected to three water regimes were generated. A genome-wide association study (GWAS) was performed for $~ 30,000$ expressed genes under each condition. In total, 73,573 eQTLs were detected, and $60 \%$ of them were resolved to a single candidate gene. Importantly, MR analysis prioritized 97 genes that contribute to drought tolerance due to gene expression variations.

\section{Results}

\section{RNA-seq analysis of $\mathbf{2 2 4}$ maize accessions subjected to three water regimes}

To understand how genetic variants affect drought-responsive gene expression on a whole transcriptome scale in maize seedlings, a collection of 224 accessions was assembled from a previously characterized population that had been used for the genetic dissection of a number of agronomic traits [17, 22-24]. These accessions were selected with the maximum retention of phenotypic diversity in regard to seedling viability under drought to achieve a wide coverage of phenotypic diversity [6] (Additional file 1: Figure S1a). At the meantime, this collection retained the genetic diversity of the original population which consists of the accessions with the tropical/subtropical (TST), temperate (including stiff stalk and non-stiff stalk), and mixed origins (Additional file 1: Figure S1b). Watering was withheld from soil-growing seedlings at the Vegetative 2-3 stage, and the decline in relative leaf water content (RLWC) was recorded. Leaf samples were collected under well-watered (WW) and the conditions when RLWC dropped to $\sim 70 \%$ (WS1) and $\sim 58 \%$ (WS2), respectively (Fig. 1a, b). The treatment and typical plant appearance under well-watered (WW), WS1, and WS2 treatments were illustrated in Fig. 1a. All samples were subjected to RNA-seq analyses. In total, 32.48 billion high-quality reads were generated, averaging 44.1 million reads per sample (Additional file 1: Figure S1c). Samples with less than 10 million reads were omitted from downstream analyses to achieve a reliable quantification of gene expression based on the unique mapped reads to the B73 reference genome RefGen_V4 [25]. As a result, 627 transcriptomes were obtained for 224 maize accessions subjected to the WW (209), WS1 (208), and WS2 (210) treatments. The single nucleotide polymorphisms (SNPs) from the yielded reads were also called for genotype confirmation and to complement previously published data on genotypic variation [16]. The average concordant rate of the genotyping data of this study and the previous work was $93.7 \%$ (Additional file 2: Table S1). To avoid any uncertainty, we designated the sites as "data missing" where inconsistent genotype data were observed. In total, 1,288,889 SNPs were obtained for the 224 maize accessions, with a missing rate $<0.6$ and a minor allele frequency $\geq 5 \%$ (Additional file 1: Figure S1d). The SNPs were later used for eQTL identification.

\section{Abscisic acid-related genes were differentially expressed in response to drought among the distinct genotypes}

Principal component analysis (PCA) was performed on the 627 transcriptomes to analyze transcriptome-wide response to different levels of water stress. Each transcriptome was represented by the expression profiles of 15,369 DEGs (genes that were differentially expressed between any of the two treatments and detected in greater than 


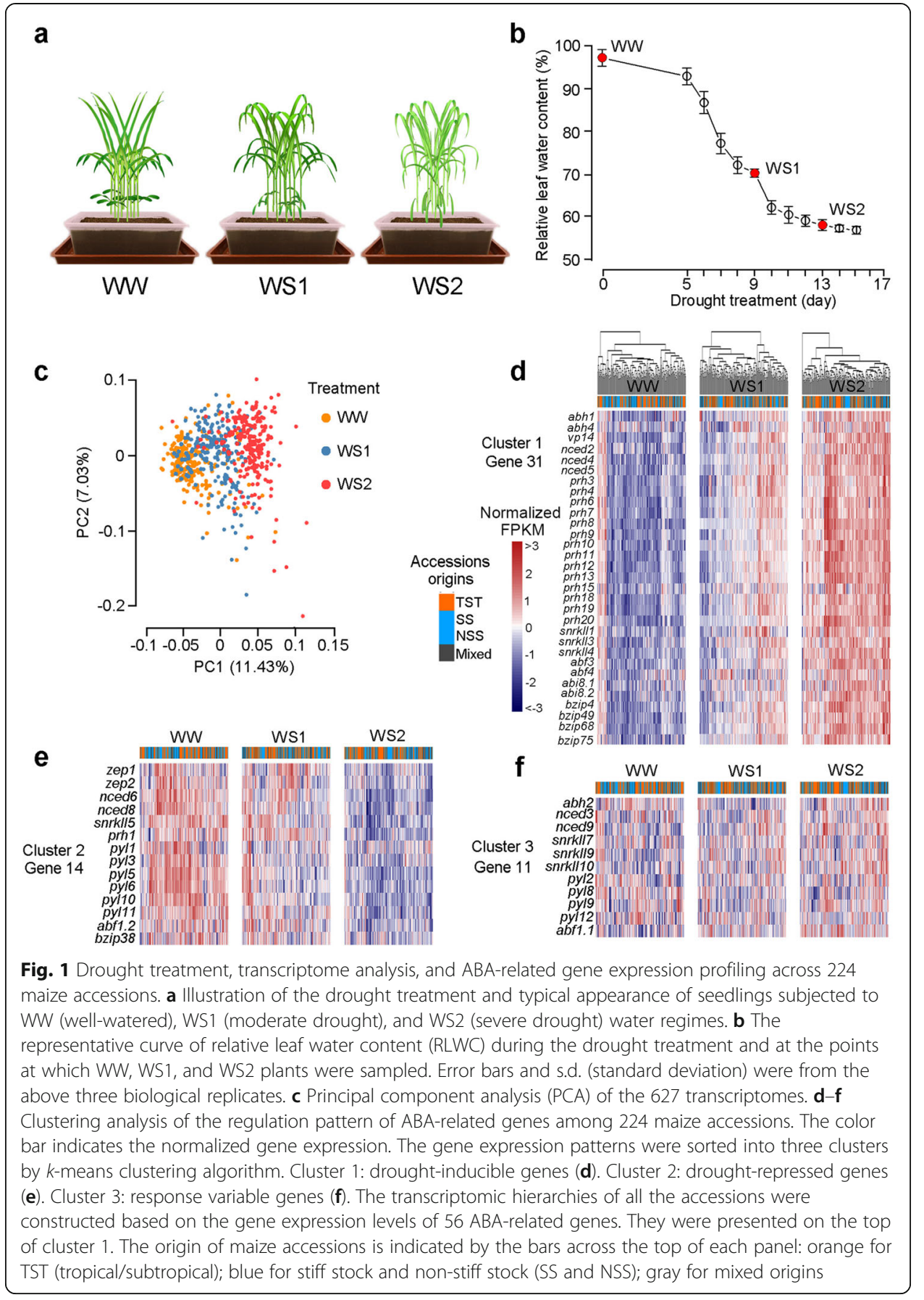

$20 \%$ of the accessions). Generally, transcriptomic changes were gradually observed with the increase in water stress. This indicates that the water stress treatments effectively induced changes in the gene expression (Fig. 1c). Using the $k$-means clustering algorithm, we classified the 15,369 DEGs into 3 clusters (Additional file 1: Figure S2a). In addition to the up- and downregulated gene clusters, 4254 genes $(27.7 \%)$ exhibited variable drought-responsive patterns among these accessions, indicating the response diversities in different genetic backgrounds. This cluster of genes was especially enriched in the Gene Ontology terms of response to various stresses or hormone stimuli, 
inferring that natural variants played a clear regulatory role on these gene expressions among the different maize accessions (Additional file 1: Figure S2b).

Since the phytohormone abscisic acid (ABA) plays a vital role in environmental stress response, we were particularly interested in gene expression variations related to ABA signaling and biosynthesis in response to the stress in the different accessions. Clustering of gene expression pattern indicated that among 56 ABA-related DEGs, 31 were generally upregulated in all the different accessions, including genes for ABA biosynthesis (vp14, nced2, nced4, and nced5), ABA catabolism (abh1 and abh4), ABA coreceptors (fourteen protein phosphate $2 C$ genes, prhs), protein kinase in ABA signaling (snrkII1, snrkII3, snrkII4), and transcription factors (bzip4, bzip49, bzip68, bizp75, abf3, abf4, abi8.1, and abi8.2) (Fig. 1d). Fourteen genes were generally downregulated by water stress, including genes encoding ABA receptors (pyl1, pyl3, pyl5, pyl6, pyl10, and pyl11); zep 1 and zep2, encoding the first enzyme in ABA biosynthesis (zeaxanthin epoxidase); and nced 6 and $n c e d 8$, encoding a rate-limiting enzyme for ABA biosynthesis (Fig. 1e). It possibly reflected a feedback of response to avoid over-reaction to the signal, which has also been observed in other phytohormone responses, such as gibberellin and brassinosteroid [26, 27]. Interestingly, eleven genes, abh2, nced3, nced9, snrkII7, snrkII9, snrkII10, pyl2, pyl8, pyl9, pyl12, and abf1.1, exhibited diversity in response direction among the investigated accessions (Fig. 1f). The diversity in response intensity and direction indicated that natural variants underlie the expression of these genes and potentially contribute to the variation of drought response among these accessions. In addition, accessions having the same origin apparently did not cluster together based on their gene expression patterns, indicating that variations in gene expression were not completely dependent on the similarity of their genomes and were also environmentally dependent (Fig. 1d-f, Additional file 1: Figure S2a).

\section{Static and dynamic eQTLs were identified in response to drought}

Expression GWAS was conducted to identify genetic variants controlling gene expressions, in which the variation in gene expression in the population was treated as a quantitative trait (etrait) and its association with the $1.28 \mathrm{M}$ genetic variants was tested. To avoid random errors, the determination of an eQTL was only recognized when at least three significant SNPs were detected in association with each treatment (Benjamini-Hochberg rejection, FDR $<0.05$ ). The identified eQTL was categorized based on the most significant SNP. Resultantly, when the start positions of the etrait gene were plotted against the position of the lead SNP of its detected eQTL, a plotted line along the diagonal was observed reflecting the local regulations (Fig. 2a). The eQTLs with lead SNPs located within $20 \mathrm{~kb}$ up- or downstream of the etrait gene were defined as local eQTLs, while all others were regarded as distant eQTLs. A comparison of the determination coefficients of the two types of eQTLs demonstrated that local eQTLs tend to have larger effects than distant eQTLs in all three water regime treatments, indicating a greater local effect on gene expression regulation (Fig. 2b). A total of 73,573 eQTLs (including 23,771 in WW, 22,945 in WS1, and 26,857 in WS2) were detected capturing an unprecedented range of regulatory variants controlling gene expression in response to drought stress (Additional file 2: Table S2). According to eQTL occurrence in different treatments, the eQTLs for an etrait that were consistently detected in all 


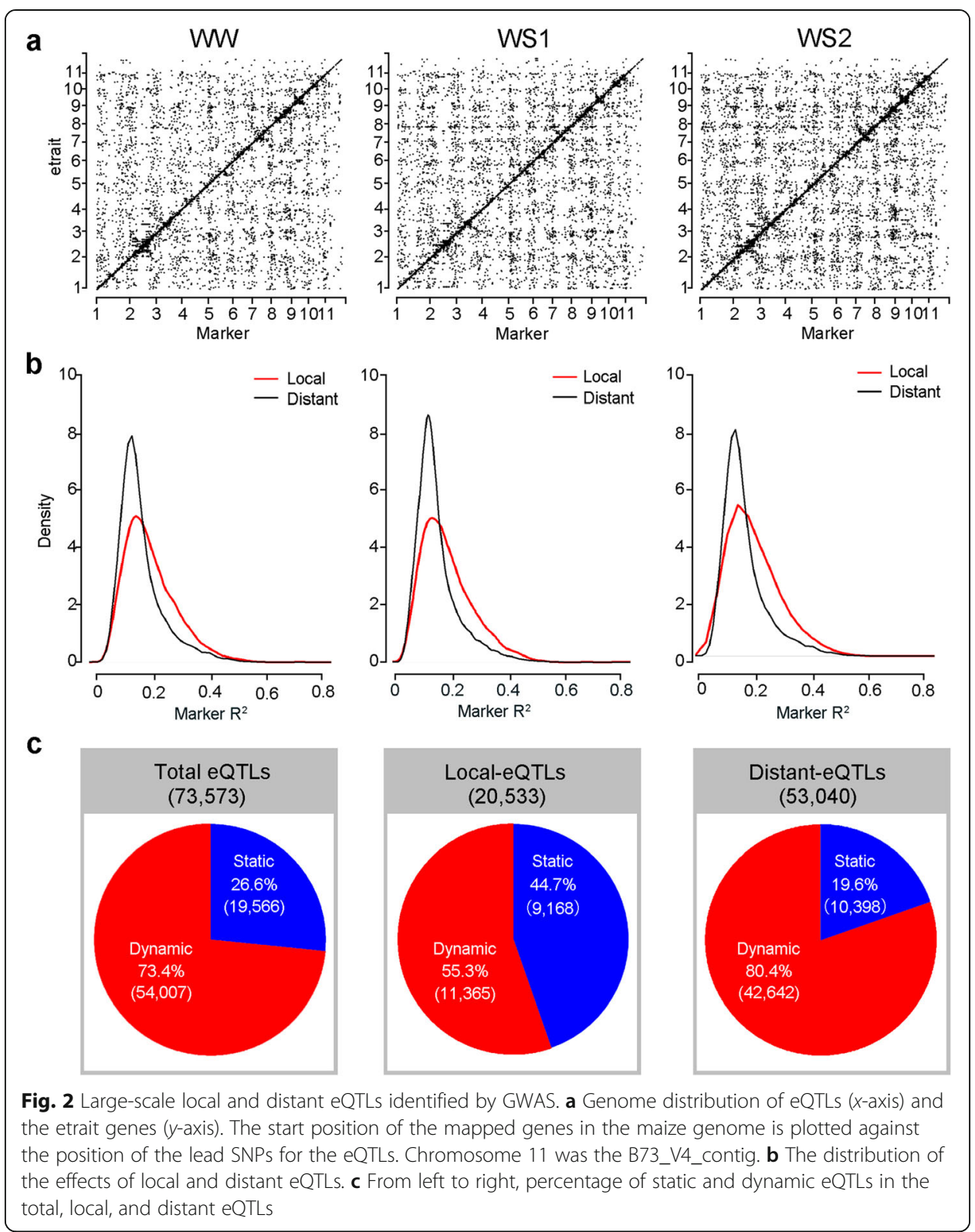

three treatments were regarded as static eQTLs, while eQTLs detected in only one or two treatments were designated as dynamic eQTLs in response to drought. Thus, 19, 566 (26.6\%) were identified as static and 54,007 (73.4\%) as dynamic eQTLs (Fig. 2c).

As for local regulatory variants, 9168 static local eQTLs (44.7\% of total 20,533 local eQTLs) were repeatedly and stably identified for 3056 genes, based on the gene expression data independently obtained from the three treatments (Fig. 2c, Additional file 2: Table S2). For example, very significant and unique association peaks were repeatedly identified in the three water regimes respectively for genes involved in plant growth and development (apc11, spiral1, oft1, cik1, rmi, arf3, iaa11, iaa26, and iaa38), signal transduction ( $c d p k 40, c a r 8$, and sinat4), potassium transport (kup3), ribosomal protein (l6), tRNA nucleoside modifier (trm10), and transcription regulation (bzip41, bzip102, $m y b 26$, and abf1) (Fig. 3a, b). The results provide strong evidence that these local 


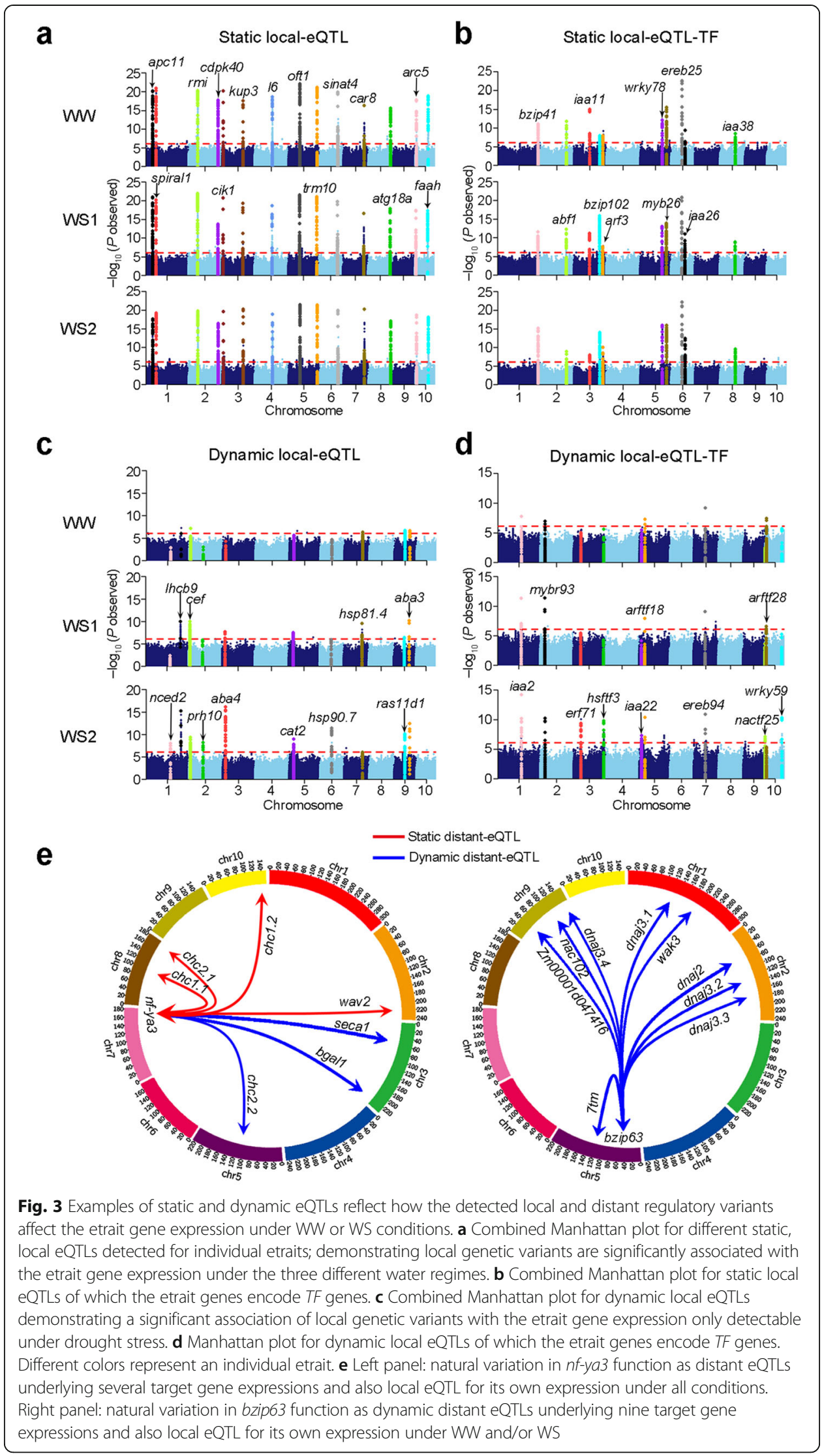


regulatory variations significantly affect their own or adjacent (within $20 \mathrm{~kb}$ ) gene expressions. Notably, among 11,365 dynamic local eQTLs, 5492 were only detected under the drought stress treatments Additional file 2: Table S2. They include genes involved in oxidative detoxification (cat2 and nactf25), photosynthesis (lhcb9), protein trafficking (ras11d1), calcium binding (cef), ABA response (nced2, prh10, aba3, and aba4), stress response (hsp81.4, hsp90.7, hsftf3, erf71, ereb94), and transcription regulation (iaa2, iaa22, arftf18, arftf28, wrky59, mybr93). The association signals were not detectable under well-watered conditions but remarkably increased upon the stress treatment, and some of them were enhanced in accordance with the increase of the stress (Fig. 3c, d). These data infer that local variants potentially confer the stress responsiveness on these genes among the different maize accessions.

As for distant regulatory variants, 10,398 static distant eQTLs and 42,642 dynamic distant eQTLs were identified, revealing a massive and dynamic regulatory network in maize seedlings (Fig. 2c, Additional file 2: Table S2). Only 19.6\% distant eQTLs were static, compared with $44.7 \%$ local eQTLs, indicating that distant genetic variants exert a more dynamic regulatory role than local eQTLs on gene expression. Several eQTL hotspots were resolved to genes encoding transcription factors (TFs). For example, a number of gene expressions were associated with natural variants in $n f-y a 3$, encoding a nuclear factor-Y TF, which plays an important role in the regulation of photoperioddependent flowering and drought stress in maize [28]. chc1.1 (Clathrin Heavy Chain1.1), chc1.2, chc2.1, and wav2 (wavy growth 2), which play important roles in stomatal movement, gas exchange, and root bending in Arabidopsis [29], were identified as $n f-y a 3$ target genes in all three water regimes (Fig. 3e). secal (chloroplast Sec translocation machinery ATPase subunit 1), which is essential for chloroplast biogenesis and the regulation of photosynthetic complexes in Arabidopsis [30], and bgal1 ( $\beta$-galactosidases 1), which contributes to the maintenance of cell wall architecture during cell elongation [31], were dynamically regulated by $n f-y a 3$ (Fig. 3e). Another example of a distant eQTL that functions as an eQTL hotspot is the bZIP TF, bzip63, which has been reported as an important node in the glucose-ABA interaction network [32]. DnaJ homologs (dnaj2, dnaj3.1, dnaj3.2, dnaj3.3, dnaj3.4), 7tm (seven-transmembrane helix), nac102, wak3 (Wall-associated kinase 3), and Zm00001d047416 were dynamically identified as target genes of bzip63. dnaj3 encodes a chaperone which has been reported to play a positive role in plant salinity and alkalinity tolerance in Arabidopsis [33].

Regulatory hierarchy based on dynamic distant eQTLs resolved to TF genes and droughtrelated target genes

One of the most plausible modes of action for distant eQTLs is the involvement of transcription factors that regulate target gene expression in a trans-acting fashion. Therefore, we were interested in the distant eQTLs resolved to TF-encoding genes. Among all 73,573 eQTLs, the lead SNPs of $3733 \mathrm{eQTLs}$ directly located in TF genes which belong to $66 \mathrm{TF}$ gene families (Additional file 2: Table S3). Gene enrichment analysis revealed that bZIP, NAC, AP2/EREBP, WRKY, and MYB were the top five TF families, indicating that their genetic variants play a major role in regulating the gene expression in maize (Additional file 1: Figure S3a). Due to our interest in the regulation of drought-responsive gene expression, we focused on the dynamic eQTLs resolved to 
TF genes and their etraits (target genes). Based on the previous knowledge on plant drought response and adaptation [34], we focused on the regulation of 44 etraits involved in different aspects of cellular response, including calcium signaling, transport, oxidative detoxification, root development, stress response, cell wall formation, and ABA signaling and biosynthesis. A drought-responsive regulatory network was constructed based on the relationship of the dynamic eQTLs (encoding TFs) and these etraits, providing a model of drought adaptation network regulated by local and distant genetic variations. A total of 45 individual TF genes, belonging to 19 TF families, were associated with 41 dynamic distant regulations of these etraits. In addition, local eQTLs were also identified for $12 T F$ genes and 26 etraits (Fig. 4, (Additional file 2: Table S4). Notably, ten of the distant regulatory activities, imparted by $M Y B, N A C, b Z I P, C 2 H 2$, and $H B$ TF family genes, were previously identified by chromatin immunoprecipitation sequencing (ChIP-seq) analysis using transgenic Arabidopsis [35], providing strong evidence for our distant eQTL identification (Additional file 2: Table S4). Since the expression of $T F$ genes can also be regulated by distant eQTLs resolved to other TFs, a regulatory hierarchy of these TF gene families can be constructed based on their regulatory relationships. As a result, $19 \mathrm{TF}$ gene families were organized into three tiers according to their hierarchical height. For example, the hierarchy position of AP2-EREBP, WRKY, and NAC families was lower than the HB and MYB families based on the ratio of the gene number of TF gene family members regulated to the number of TFs that regulate them. A similar regulatory hierarchy was also detected in studies of Arabidopsis and Populus $[35,36]$. Regulatory relationships were also identified for some less-reported, drought-related TFs, such as TCP, C3H, and AUX/IAA, which provides valuable

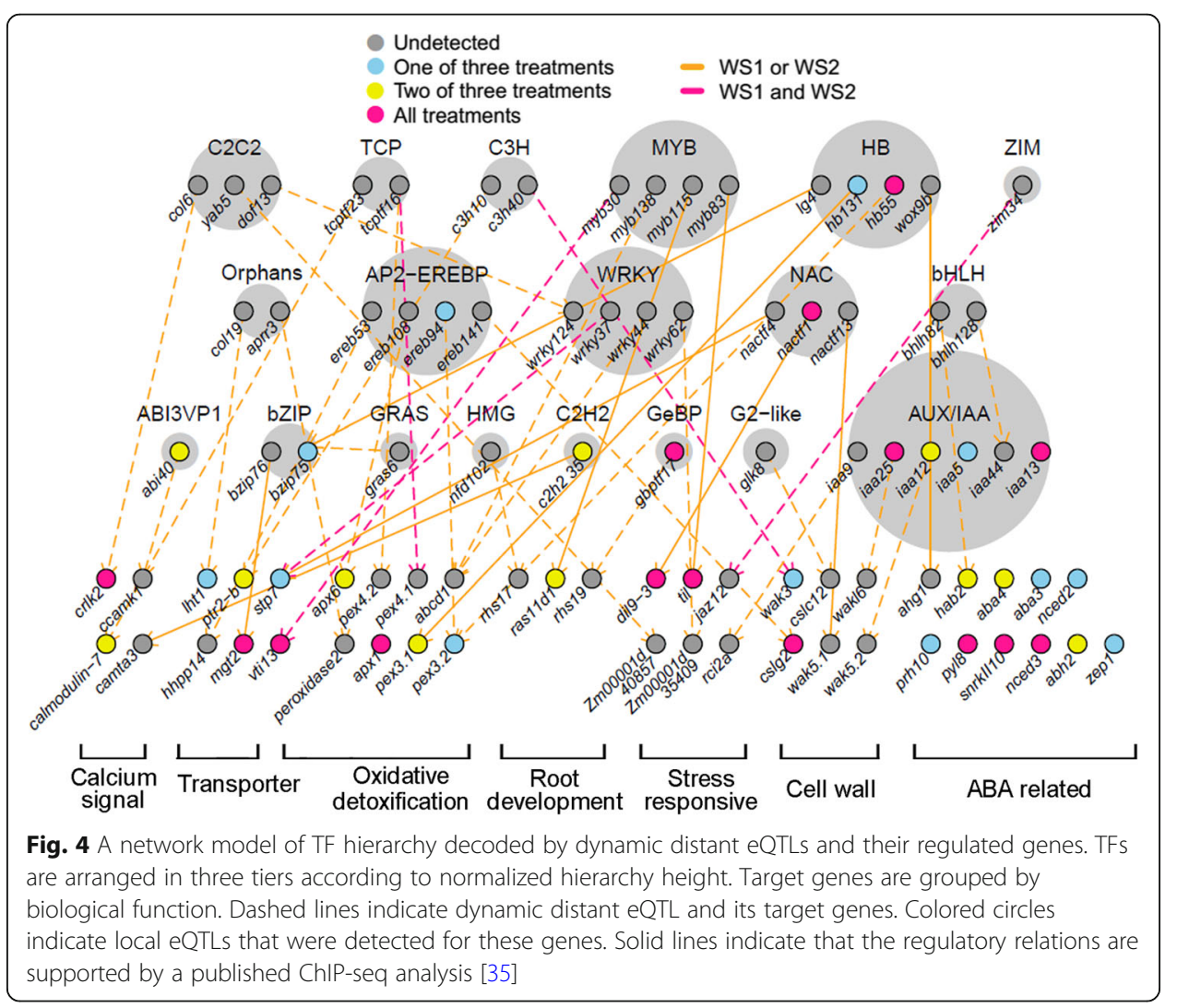


information for further studies. For example, two tandem Peroxin genes, pex4.1 and pex4.2, are potentially regulated by tcptf16, a TCP family TF. The expression of two Wallassociated kinase, wak5.2 and wakl6, may be controlled by two IAA TFs (Fig. 4).

\section{Mendelian randomization test prioritized 97 genes associated with drought tolerance}

Based on the newly obtained data on gene expression and the previous phenotypic measurements of drought tolerance of these accessions [6], we performed Mendelian randomization (MR) test [21] to identify genes whose expression levels (with identified regulatory variants) are relevant to plant drought tolerance. As a result, 97 candidate genes were prioritized for their contribution to drought tolerance based on their alterations in gene expression (Bonferroni threshold, $P<3.33 \times 10^{-5}$ ). The expression of 51 genes was found to positively contribute to drought tolerance, and 46 were negatively associated with the trait (Fig. 5a, Additional file 2: Table S5). The positive regulators were identified for plant viability because their expression variations were positively related to plant survival rate under drought (Fig. 5b). Among them, ZmVPP1 gene expression was found to be regulated by a dynamic local eQTL, and the expression was positively related with drought tolerance, which was consistent with the result from previous GWAS for drought-tolerant gene in maize seedling [6], supporting the present analytic strategy and statistical power of the MR analysis (Fig. 5a, Additional file 2: Table S5). Other identified positive regulators for drought tolerance (Fig. 5b) include the following: Zm00001d031323 (csn6a) is a homologoue of Arabidopsis CSN6A (COP9 signalosome subunit 6A) which plays pleiotropic roles in plant development by regulating ubiquitination-mediated protein degradation [37]; Zm00001d031790 (abf4), a homolog of Arabidopsis ABF4, which plays an important role in drought stress- and ABA-responsive gene expression [38]; Zm00001d032346 and Zm00001d019946 (uge5.1 and uge5.2) are paralogous genes encoding UDP-D-glucose 4-epimerase (UGE) which interconverts UDP-glucose and UDP-galactose. UGEs function in cell wall carbohydrate biosynthesis and possibly in stress tolerance by supplying UDP-galactose for galactinol biosynthesis which was considered to act as an osmoprotectant in abiotic stress tolerance in Arabidopsis [39]; and Zm00001d047517 encoding a reticulon-like protein, and its homolog in mammals was reported functioning in cell apoptosis and development [40].

As for the negative regulators, the regulatory variants and gene expression levels of the following genes were exemplified that they were negatively associated with plant drought tolerance (Fig. 5b, Additional file 2: Table S5). Zm00001d051554 (abh2) encodes an abscisic acid 8'-hydroxylase (ABAox), which catalyzes the first step in the oxidative degradation of ABA, and in Arabidopsis, it promotes stomatal opening in response to high humidity [41]. Zm00001d043902 (asil2) encodes a trihelix-transcription factor, and the Arabidopsis homolog represses seed maturation and desiccation-tolerant gene expression in vegetative tissues [42]. Zm00001d017378 (rma1) encodes an E3 ubiquitin-protein ligase, similar to Arabidopsis RMA1, which plays a critical role in the proteolysis of plasma membrane aquaporin in response to dehydration [43]. Zm00001d009365 (sweet2) encodes a putative sugar transporter, and its homologs in Arabidopsis and rice are characterized as hexose transporters on tonoplasts that are responsible for the transport of excess glucose from the cytosol into vacuoles [44]. All the prioritized genes represent valuable candidates for future functional validation and allelic mining. 


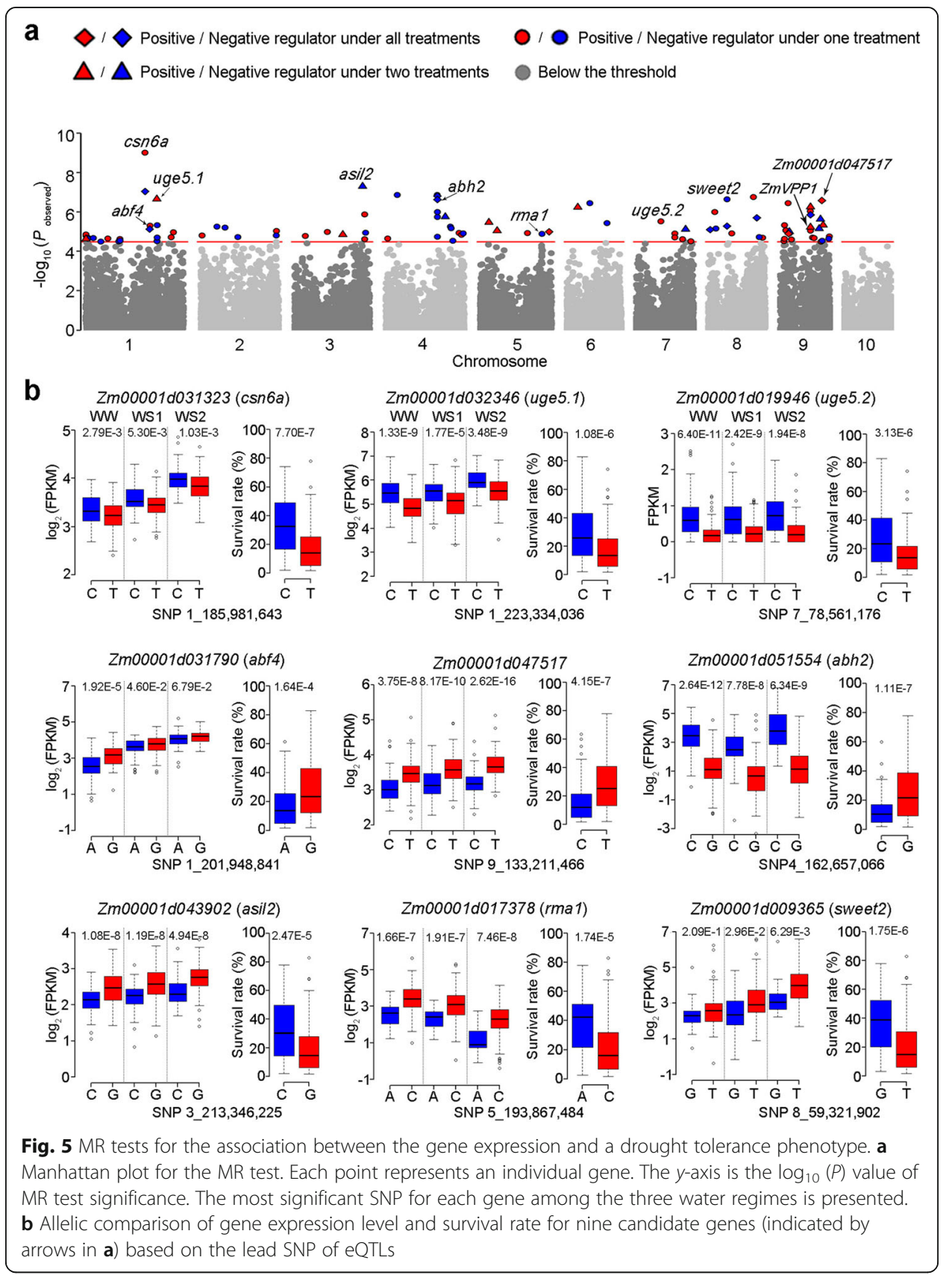

Local variants regulate $a b h 2$ expression and negatively associated with drought tolerance

To verify the abovementioned findings, a $3.5-\mathrm{kb}$ genomic region of abh2 was sequenced among 141 maize inbred lines to check whether abh2 expression levels were affected by local regulatory variants, and its expression was negatively related with drought tolerance (Fig. 5a, Additional file 2: Table S5). In total, 265 SNPs and 76 insertions or deletions (InDels) were identified, and all the significant variants $\left(P<1 \times 10^{-6}\right)$ were synonymous or locating in non-coding regions (Fig. 6a). Six major haplotype groups were identified based on significant variants $\left(P<1 \times 10^{-6}\right.$, accession number $>5$, Fig. $\left.6 \mathrm{~b}\right)$. Haplotype 1 containing SNP2344 (C-genotype), the lead SNP identified as the abh2 local eQTL, had 


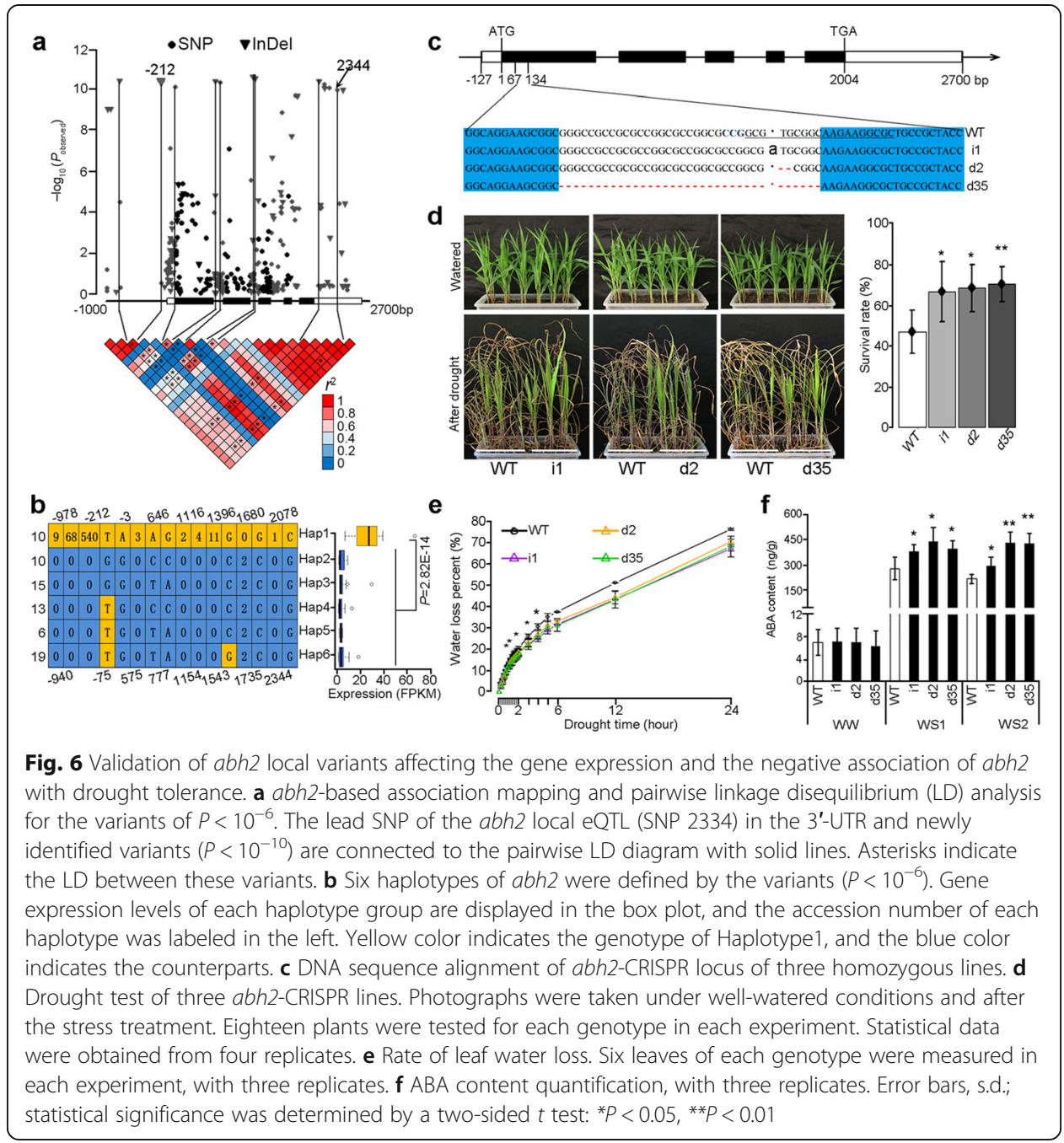

significantly higher $a b h 2$ gene expression than other haplotypes, supporting that local genetic variants affect the $a b h 2$ expression (Fig. 5b). Since $a b h 2$ expression was identified to be negatively associated with plant drought tolerance, the Clustered Regularly Interspaced Short Palindromic Repeats (CRISPR)-Cas9 technology was employed to generate the ahb2-CRISPR knockout plants. Three independent homozygous lines (i1, d2, and d35) were obtained (Fig. 6c), and their drought tolerance was compared with wild-type plants. The viability of all three mutants was significantly higher than wild-type plants after drought, supporting a negative role of $a b h 2$ in stress tolerance (Fig. $6 \mathrm{~d}$ ). Less water loss was observed in detached leaves of the abh2-CRISPR lines over the course of a 1- to 4-h dehydration treatment, indicating that the abh2 knockout potentially conferred a quicker stomatal closure in response to dehydration stress (Fig. 6e). When ABA content was quantified, the three CRIPSR lines exhibited approximately a 30\% increase in ABA content compared to the wild type in the WS1 and WS2 treatments, suggesting that interfering abh2 function may sustain a higher level of ABA when plants were challenged by stress (Fig. 6f). Collectively, the results support the implications of the eQTL and MR analyses. The identified natural variation in $a b h 2$ provides a favorable allele for plant drought tolerance by modulating ABA content in response to water deficit. 


\section{Discussion}

In the present study, 73,573 eQTLs (regulatory variants) were identified that are responsible for the variation in the expression of $\sim 30,000$ genes during drought response. The degree of LD is a major factor affecting the resolution of association mapping. The genome-wide LD decay $\left(r^{2}<0.2\right)$ in maize is $\sim 5.5 \mathrm{~kb}$ in maize Hapmap 2 [3] and was estimated to be $1.6 \mathrm{~kb}$ in the association panel in the present study, based on the integration of 1.28M SNPs (Additional file 1: Figure S3b). Owing to the rapid LD decay, which confers high mapping resolution, over $60 \%$ of the eQTLs attained a resolution level of one candidate gene. About $20 \%$ of the eQTLs were resolved to two candidate genes. In a comparison of the eQTLs detected in the present study with those identified in maize kernels (15 days after pollination [16]), only about $12 \%$ of the eQTLs overlapped, indicating that gene expression architecture is tissue-dependent and dynamic (Additional file 1: Figure S3c). Approximately 27\% of the eQTLs, within the identified 73,573 eQTLs, were static and consistently identified across the three conditions. This provides strong evidence on how these genes are genetically regulated. Notably, 53,040 dynamic eQTLs were identified, capturing an unprecedented number of regulatory variants for drought response in maize seedlings and providing an important foundation for future analyses. Although the decision was made that at least three significant SNPs needed to be detected to identify and designate an eQTL, this does not fully rule out the potential effect of random errors in the quantification of gene expression and result in the overestimation of the number of dynamic eQTLs. Therefore, we recommend that dynamic eQTLs detected under both WS1 and WS2, but not under WW, should be prioritized for subsequent studies pertaining to the regulation of drought-responsive gene expression. Lead SNPs of dynamic local eQTLs were more frequently located in regulatory regions of the candidate gene compared with the lead SNPs in static eQTLs, indicating that stress-responsive sequences are potentially detectable in the up- or downstream of genes associated with dynamic local eQTLs (Additional file 1: Figure S3d).

Distant eQTLs usually act through diffusible factors to fulfill long-range control of gene transcription. In particular, transcription factors serve classic roles as regulatory agents that function as molecular switches of the gene expression at the terminal end of the stress signal transduction pathways associated with stress response [45]. A total of 3733 distant eQTLs were resolved to transcription factors, and a multi-level and multi-family model of a transcriptional regulation hierarchy was constructed based on their regulatory relationships (Fig. 4, Additional file 2: Table S4). To our knowledge, this represents the first comprehensive regulatory network constructed through eQTL analysis, owing to the high resolution of candidate gene detection by GWAS in maize. Previously, the target genes of a given TF were usually identified through analysis of specific gene overexpression or mutant lines and then possibly confirmed by ChIP$\mathrm{qPCR} / \mathrm{seq}$ analysis. Although the strategy and results are highly informative, the throughput using this approach is rather low, and more importantly, the interactive or orchestral effects of different types of TFs in an organism cannot be simultaneously considered and evaluated.

An eQTL hotspot was detected on chromosome 6, which is associated with the expression variation of a cluster of 21-24 nearby genes in response to the three water regimes. Notably, this hotspot and the regulated genes were located within a $2.9-\mathrm{Mb}$ 
presence-absence variation (B73_V4_Chr6: 22.4-25.3 Mb) between the B73 and Mo17 genome [46]. Another eQTL hotspot (B73_V4_Chr2: 120.0-153.1 Mb) was rich in structural variations [47], and the LD in this region was significantly greater than other regions (Additional file 1: Figure S4), indicating that structural variation is an important resource for regional-level LD and expression variation. The growing availability of whole-genome sequences of additional maize accessions will undoubtedly facilitate a better understanding of the nature of the causal polymorphisms underlining eQTLs and eQTL hotspots.

eQTL mapping combined with MR analysis facilitates bridging the gap between genetic and phenotypic associations. In the present study, MR analysis prioritized 97 candidate genes in the regulation of drought response; including 51 positive and 46 negative contributors (regarding gene expression) to maize drought tolerance (Fig. 5, Additional file 2: Table S5). Among them, ahb2 was verified negatively associated with drought tolerance in maize seedlings. Re-sequencing of the $3.5-\mathrm{kb}$ abh2 genomic fragment among 141 accessions identified InDel-212 (540 bp in length) which was significantly associated with the gene expression and strong LD with the eQTL lead SNP (SNP2344) (Fig. 6a). InDel-212 was identified as a DTA (hAT) family DNA transposon element flanked by an 8-bp target site duplication [48]. It is located 212 bp upstream of the start codon (ATG) and contains one MYB (TGGTTAG) and one MYC binding element (CAATTG), according to the plant cis-acting regulatory DNA elements (https://www.dna.affrc.go.jp/PLACE/). Consequently, the presence of these regulatory elements provides evidence to suggest that InDel-212 may play an important role in the regulation of gene expression (Additional file 1: Figure S5a). Further exploration of the 9-kb abh2 locus between B73 and CIMBL55, a drought-tolerant accession [6, 10], unraveled two additional structure variations. However, when they were genotyped among the 141 accessions, none of them was more significantly associated with the gene expression than InDel-212 under drought stress (Additional file 1: Figure S5b). In addition, several RNAPII- and H3K4me3 chromatin interaction loops were detected in close proximity to InDel-212, based on the chromatin interaction analysis by pairedend tag (ChIA-PET) sequencing in B73 [49]. Taken together, these data collectively suggest that InDel-212, a 540-bp insertion present in drought-susceptible accessions, may be the causative variation of $a b h 2$.

ABA is involved in a variety of physiological processes during the plant life cycle, including seed dormancy, germination, stomatal movement, fruit development, and responses to biotic and abiotic stresses [45,50]. A phylogenetic analysis revealed that in several plant species (Arabidopsis, rice, and sorghum), ABA 8'-hydroxylase is encoded by multiple genes (Additional file 1: Figure S6a). There are five paralogous genes in the maize genome, of which the two closest homologs, abh1 and abh2, exhibit a similar pattern of tissue expression (Additional file 1: Figure S6b). None of the three knockout alleles of $a b h 2$ displayed any obvious phenotypic abnormalities in regard to the germination or plant development, probably due to the functional redundancy or compensation provided by the paralogous genes. Although modulating endogenous ABA level or response has been supposed to be a promising strategy for plant stress tolerance improvement, it is still challenging to determine, among a number of ABA-related genes, which gene and genetic modification strategy are appropriate to enhance the trait. The present work represents a promising target and strategy. Disrupting abh2 function or 
reducing its expression can fine-tune $\mathrm{ABA}$ levels in plants subjected to drought for the genetic improvement of plant stress tolerance.

\section{Conclusions}

The regulatory variants controlling the gene expression, constitutively or drought dynamically, were unraveled on a genome-wide scale in maize. It provides significant information that can be used to better understand the role of distal and proximal genetic effects on gene expression regulation. The prioritized candidate genes in drought tolerance are valuable targets for further gene functional study or allelic mining. Gene knockout strategy may be directly applied to genes whose expression is negatively associated with stress tolerance to improve stress tolerance.

\section{Methods}

\section{Plant accessions and RNA-seq analysis}

Two hundred twenty-four maize accessions (inbred lines) were derived from a maize association mapping panel $[6,17]$, including three additional lines (A188, W22, and X178). Plants were germinated and cultivated as previously described [6]. Three duplicated cultivations were performed for each accession. When the seedlings were 2 weeks old, drought treatment was applied to two duplicated cultivations by withholding water, while keeping one duplication for normal growth. On the ninth and thirteenth days of drought treatment, samples of WS1 and WS2 were harvested, respectively. Samples of WW were harvested on the same day with WS1. WS2 were sampled on a similar time point of a day to avoid possible circadian difference in gene expressions 4 days later. Five-cm leaf sections in the middle of the second leaves were harvested from three plants of each germplasm, pooled, and frozen in liquid nitrogen. The samples were stored at $-80^{\circ} \mathrm{C}$ prior to RNA extraction. Total RNA was isolated using TRIzol reagent (Biotopped). Libraries from the resulting total RNA were prepared using the TruSeq paired-end mRNA-Seq kit and protocol from Illumina and sequenced on the Illumina HiSequation 2500 system with Illumina TruSeq SBS v3 reagents as previously described [51]. Fifty-eight biological replicates were randomly placed in the different treatments to estimate the repeatability of the experimental procedures. The average expression correlation coefficient of the biological replicates was 0.83 , indicating a reasonable reproducibility of the extensive sampling and expression quantification process.

\section{Sequencing reads quality control and mapping}

After removing reads with low sequencing quality and reads with sequencing adapter, we filtered those samples with sequenced reads number less than 10 million to avoid inaccurate quantitative analysis of unsaturated sequencing. Six hundred twenty-seven high-quality RNA-seq samples of 224 inbred lines under three drought treatments were obtained for the following analysis (WW, $n=209$; WS1, $n=208$; WS2, $n=210$ ), with additional 58 random biological repeats. All sequenced high-quality reads were mapped to the B73_RefGen_v4 (ftp://ftp.ensemblgenomes.org/pub/plants/release-38/fasta/zea_ mays/dna/) maize genome using HISAT2 ver.2.0.5 (with parameters: --dta; --score-min

L,-0.6,-0.6) [52, 53]. Finally, only alignments with mapping quality (MQ) $>25$ for 
single-end reads and concordant unique alignments for paired-end reads were kept for further analysis.

\section{Gene expression quantification and clustering analysis}

All mapped reads with $\mathrm{MQ}>25$ were sorted according to their alignment position on the genome. Each gene was calculated by featureCounts [54], and the differentially expressed genes among different germplasms were identified by the edgeR package [55]. Furthermore, the genes with significant differences in at least $20 \%$ of germplasm between any two treatments (false discovery rate (FDR) $<0.05)$ were regarded as differentially expressed genes (DEGs). The StringTie ver.1.3.4d (with parameters: -G -e -B -o -A) $[52,56]$ software was used to quantify the gene expression levels by calculating the fragments per kilobase of exon per million fragments mapped (FPKM) to normalize the sequencing depth and gene length. To eliminate the batch effects and systematic variation caused by experimental technique, we normalized the FPKM of all expressed genes of each sample by normalizeQuantiles function of LIMMA package in R. Principal component analysis were performed with the prcomp function in $\mathrm{R}$ with setting scale $=$ TRUE. According to the elbow method algorithm, the expression matrix was drawn through the pheatmap package with kmeans_k $=3$. We normalized the FPKM values of each gene using a normal quantile transformation (qqnorm function in R). Those genes with normalized FPKM above 0.05 across at least $20 \%$ of the accessions were computed for eQTLs detection by a mixed linear model.

\section{SNP detection and filtering}

To identify the genotype of each inbred line, we merged the alignment results (MQ > 25) of three different treatments for each germplasm into one file in bam format and then detected SNPs using the Genome Analysis Toolkit (GATK, version 4.0.5.0) [57]. Firstly, we marked those duplicated reads with the Picard package v1.115 MarkDuplicates (http://broadinstitute.github.io/picard/) and filtered out the spliced mapped reads using SplitNCigarReads package of GATK. Secondly, we performed SNP variant calling by both HaplotypeCaller package of GATK and mpileup package of SAMtools [57, 58], and then we defined the credible variants as those intersection variants that satisfied the following parameters: quality depth (QD) $\geq 2.0$ and ReadPosRankSum $\geq-8.0$ and FS $\leq 60.0$ and Qual $\geq$ meanQual. Thirdly, we recalibrated the base quality score based on our defined credible variants using the BaseRecalibrator package of GATK. Lastly, we performed the final SNP variant calling by the HaplotypeCaller and GenotypeGVCFs package of GATK. All detected bi-allelic SNP variants were used for further analysis.

To obtain a high-quality genotype, we firstly filtered out those low-quality SNPs by the GATK VariantFiltration package with the following parameters: --cluster-windowsize 35, --cluster-size 3, FS $\leq 30$, and $\mathrm{QD} \geq 2.0$. For each germplasm, if the $\mathrm{MAF}<0.05$, we considered that it was due to sequencing errors and processed the heterozygous genotypes by modifying the genotype of the minor allele, into its corresponding major allele. Whereas we masked this heterozygous position as missing data. Finally, 1,899,223 SNPs were obtained from our sequenced dataset. To estimate the accuracy of the genotype called from this study, we download another high-quality SNPs dataset derived 
from 368 inbred lines which is consisting of 1.25M SNPs with a minor allele frequency greater than 0.05 (http://www.maizego.org/Resources.html) [16] and calculated the concordant rate of the genotypes by comparing the overlapping SNP set from the same inbred line. We further integrated our genotype dataset with the $1.25 \mathrm{M}$ dataset to increase the SNP maker density, and 2,912,412 SNPs were obtained. After filtering SNPs with missing rate $<0.6$ and MAF $\geq 0.05$, finally, 1,288,889 SNPs were retained and used for further association analysis.

\section{GWAS for eQTL identification}

eQTL analysis for each expressed gene, whose expression level (FPKM) was above 0.05 in more than 20\% accessions (29,614 genes in WW, 30,019 genes in WS1, 30,272 genes in WS2), was computed for GWAS analysis using a linear mixed model [59] by incorporating the kinship coefficients, population structure, and hidden confounding factors, performed by TASSEL (version 5.0) with -mlm -mlmVarCompEst P3D -mlmCompressionLevel None.

The kinship coefficients were estimated by selecting 81,612 uniformly distributed SNPs which was of the lowest missing rate in 10-kb windows, using PHYLIP (version 3.696). The population structure was inferred with fastSTRUCTURE [60]. We further estimated the hidden confounding factors contributing to the expression variability by Bayesian factor analysis (implemented in PEER), and fourteen confounding hidden factors accounting for gene expression variability of each drought treatment were retained and included in the mixed model to examine the validity of association significance, respectively [61, 62]. Benjamini-Hochberg $(\mathrm{BH})$ rejection thresholds were set for WW, $P<7.64 \mathrm{E}-7$; WS1, $P<7.20 \mathrm{E}-7$; and WS2, $P<8.36 \mathrm{E}-7$ (FDR $<0.05$ ). The eQTLs for a specific etrait were identified through three consecutive steps: (1) we grouped all significant SNPs into one eQTL, if their physical distance is $<5 \mathrm{~kb}$; (2) if the SNPs from different eQTLs were within a LD block $\left(r^{2}>0.1\right)$, calculated by Haploview with the parameters: -n -maxdistance 250 -minMAF 0.05 -hwcutoff 0 -dprime [63], the eQTLs were combined and the most significant SNP was retained; (3) if multiple eQTLs were detected within one candidate regulatory gene, they were combined and the most significant SNP was retained. Finally, the most significant SNP (lead SNP) was representative of the detected eQTL.

\section{Regulatory hierarchy network inference}

The entire set of available characterized maize transcription factors (www.grassius.org/ grasstfdb.php and http://plntfdb.bio.uni-potsdam.de/v3.0/) was used to identify certain TF families. The hierarchy height of TFs was calculated as previously described: $h=(O$ $-I) /(O+I)$, where $O$ and $I$ are out-degree and in-degree of examined TF through regulatory relationships, respectively, as described [35].

\section{Mendelian randomization analysis}

MR analysis was performed to reveal the relationship between gene expression level and the phenotype as previously described [21]. In detail, $z$ was denoted as the most significantly associated SNP detected by the eQTL analysis under WW, WS1, and WS2 conditions; $x$ as the normalized gene expression levels; and $y$ as 
the survival rate of maize seedlings under drought [6]. Then, a two-step leastsquares (2SLS) estimate of the effect of $x$ on $y$ from an MR analysis was $b_{x y}=b_{z y} /$ $b_{z x}$, where $b_{z y}$ and $b_{z x}$ were the least-squares estimates of $y$ and $x$ on $z$, respectively, and $b_{x y}$ was interpreted as the effect size of $x$ on $y$ free of confounding from non-genetic factors. The sampling variance of the 2SLS estimate was var. $\left(b_{x y}\right)=[$ $\left.\operatorname{var}(y)\left(1-R_{x y}^{2}\right)\right] /\left[n \operatorname{var}(x) R_{z x}^{2}\right]$, where $n$ was the sample size, $R_{x y}^{2}$ was the proportion of variance in $y$ explained by $x$, and $R_{z x}^{2}$ was the proportion of variance in $x$ explained by $z$. We therefore could have a statistic $T_{\mathrm{MR}}=b_{x y}^{2} /$ var. $\left(b_{x y}\right)$ to test the significance of $b_{x y}$, where $T_{\mathrm{MR}}=X_{1}^{2}$. In total, 30,006 tests (genes with identified eQTLs on the same chromosome) were done, therefore, we set a uniformed experiment-wise significance Bonferroni threshold as $P<3.33 \times 10^{-5}$.

\section{Generation and analysis of abh2-CRISPR lines}

The CRISPR/Cas9 knockout vector was constructed according to the previously described [64]. The Oligo-F and Oligo- $\mathrm{R}$ were annealed and inserted between two BsaI sites of pBUE411. The resulting vector was transformed into Agrobacterium strain EHA105, then the Agrobacterium-mediated method was used to transform maize immature embryos. Three $\mathrm{T}_{1}$ plants with a homozygous mutation at the target sites were self-pollinated to get $T_{2}$ progenies. $T_{2}$ plants were further genotyped by PCR and sequencing to confirm the presence of mutations at the target sites (Additional file 2: Table S6). $\mathrm{T}_{2}$ and wild-type plants were used for further analyses. Drought test was performed as described by Wang et al. [6]. At least 18 plants of each line were compared in each test, and statistical analyses were based on the data obtained from four independent experiments. Leaf water loss was determined by a gravimetric method. The second leaves were harvested from three plants of each genotype at the V2 stage. Leaf water loss was determined by allowing detached leaves to desiccate at room temperature $\left(25^{\circ} \mathrm{C}\right)$ and humidity (48\%) and weighing every $10 \mathrm{~min}$ for the first $2 \mathrm{~h}$, and once an hour to $24 \mathrm{~h}$. Leaf water loss rate was calculated based on gravimetric water loss in relation to fresh weight from three biological replicates.

\section{Quantification of endogenous ABA content}

The planting and drought treatment methods are the same as those of RNA sequencing samples. Five-cm-long leaf sections in the middle of the second leaves were harvested from three plants of each genotype, pooled, and flash-frozen in liquid nitrogen. Fifty milligrams of the fine powder was mixed with $500 \mu \mathrm{l}$ extracting solution (IPA: $\mathrm{H}_{2} \mathrm{O}: \mathrm{HCl}=1000: 500: 1$ ) and $50 \mu$ internal standard solution (containing $10 \mathrm{ng} \mathrm{C}_{15} \mathrm{H}_{14} \mathrm{D}_{6} \mathrm{O}_{4}$ ) and in a shaking incubation at $4{ }^{\circ} \mathrm{C}$ for $0.5 \mathrm{~h}$ in $1.5 \mathrm{ml}$ centrifuge tubes. Then, $1 \mathrm{ml}$ of trichloromethane $\left(\mathrm{CHCl}_{3}\right)$ was added and mixed at $4{ }^{\circ} \mathrm{C}$ for $0.5 \mathrm{~h}$. The bottom solution $(\sim 1.2 \mathrm{ml})$ obtained by centrifugation was blowdried with nitrogen gas. Dissolved with $100 \mu \mathrm{l}$ methanol, the supernatant after centrifugation was determined by UPLC-Q Extractive MS in biomass spectrometry laboratory, China Agricultural University (Beijing, China). 


\section{Supplementary information}

Supplementary information accompanies this paper at https://doi.org/10.1186/s13059-020-02069-1.

Additional file 1: Figure S1. Population characterization of 224 maize accessions and the number of RNA-seq reads. Figure S2. Expression pattern clustering of 15,369 differentially expressed genes (DEGs) in response to drought stress and gene ontology enrichment of each cluster. Figure S3. Statistical analysis of dynamic-eQTLs encoding TF genes, estimation of the genome-wide linkage disequilibrium (LD) decay, comparison of eQTLs detected in maize kernels and leaves, and the distribution of the lead SNPs of the static and dynamic local-eQTLs. Figure S4. Comparison of hotspots, structural variation distribution, and LD on maize chromosomes. Figure S5. Analysis of the causative variation of abh2. Figure S6. Phylogenetic and expression level comparison of abh2 homologous genes.

Additional file 2: Table S1. The SNP concordant rate between the previous and present studies. Table S2. Information on static and dynamic eQTLs identified in the present study. Table S3. 3733 eQTLs directly located in TF genes. Table S4. Detail information on the eQTL network. Table S5. Detail information on 97 candidate genes identified by Mendelian Randomization analysis. Table S6. Primers used in the present study.

Additional file 3. Review history.

\section{Acknowledgements}

We appreciated the excellent technical assistance in maize transformation provided by the Center for Crop Functional Genomics and Molecular Breeding in China Agricultural University.

\section{Peer review information}

Yixin Yao was the primary editor of this article and managed its editorial process and peer review in collaboration with the rest of the editorial team.

\section{Review history}

The review history is available as Additional file 3.

\section{Authors' contributions}

SL, LC, and WH performed the experiments, analyzed the data, and drafted the manuscript. SW, SY, and XL assisted in the sequencing analysis of abh2 and the drought tolerance test of abh2-CRISPR lines. JY provided the materials and helped in the project design. BL, MB, and GZ-H performed the RNA-seq experiments. SS advised on the eQTL analysis and revised the manuscript. FQ conceived and advised on the experiments and revised the manuscript. The author(s) read and approved the final manuscript.

\section{Funding}

This research was supported by the Beijing Outstanding Young Scientist Program (BJJWZYJH01201910019026), the National Key Research and Development Plan of China (2016YFD0100605), the National Natural Science Foundation of China (31625022), and the Youth Innovation Promotion Association of Chinese Academy of Science (2017141).

\section{Availability of data and materials}

The RNA-seq reads, gene expression level (FPKM) in each sample, and sequence variation data generated in this study have been deposited to the Genome Sequence Archive [65] and Genome Variation Map [66] in BIG Data Center [67], Beijing Institute of Genomics (BIG), Chinese Academy of Science, under accession number (CRA000334 [68], CRA002002 [69] and GVM000048 [70]). The script for the MR analysis can be accessed at GitHub [71]. The RNA-seq reads also have been deposited in the National Center for Biotechnology Information under project number PRJNA637522 [72].

Ethics approval and consent to participate

Not applicable.

\section{Consent for publication}

Not applicable

\section{Competing interests}

The authors declare no competing interests.

\section{Author details}

'State Key Laboratory of Plant Physiology and Biochemistry, College of Biological Sciences, China Agricultural University, Beijing 100193, China. ${ }^{2}$ National Genomics Data Center \& CAS Key Laboratory of Genome Sciences and Information, Beijing Institute of Genomics, Chinese Academy of Sciences, and China National Center for Bioinformation, Beijing 100101, China. ${ }^{3}$ Agricultural College, Yangtze University, Jingzhou 434025, China. ${ }^{4}$ National Key Laboratory of Crop Genetic Improvement, Huazhong Agricultural University, Wuhan 430070, China. ${ }^{5}$ Corteva Agriscience, Johnston, IA 50131, USA. 
Received: 12 March 2020 Accepted: 10 June 2020

Published online: 06 July 2020

\section{References}

1. Godfray HC, Beddington JR, Crute IR, Haddad L, Lawrence D, Muir JF, Pretty J, Robinson S, Thomas SM, Toulmin C. Food security: the challenge of feeding 9 billion people. Science. 2010;327:812-8.

2. Andorf C, Beavis WD, Hufford M, Smith S, Suza WP, Wang K, Woodhouse M, Yu J, Lubberstedt T. Technological advances in maize breeding: past, present and future. Theor Appl Genet. 2019;132:817-49.

3. Chia JM, Song C, Bradbury PJ, Costich D, de Leon N, Doebley J, Elshire RJ, Gaut B, Geller L, Glaubitz JC, et al. Maize HapMap2 identifies extant variation from a genome in flux. Nat Genet. 2012;44:803-7.

4. Lobell DB, Roberts MJ, Schlenker W, Braun N, Little BB, Rejesus RM, Hammer GL. Greater sensitivity to drought accompanies maize yield increase in the U.S. Midwest. Science. 2014;344:516-9.

5. Yang W, Guo Z, Huang C, Duan L, Chen G, Jiang N, Fang W, Feng H, Xie W, Lian X, et al. Combining high-throughput phenotyping and genome-wide association studies to reveal natural genetic variation in rice. Nat Commun. 2014;5:5087.

6. Wang X, Wang H, Liu S, Ferjani A, Li J, Yan J, Yang X, Qin F. Genetic variation in ZmVPP1 contributes to drought tolerance in maize seedlings. Nat Genet. 2016;48:1233-41.

7. Jang HS, Shah NM, Du AY, Dailey ZZ, Pehrsson EC, Godoy PM, Zhang D, Li D, Xing X, Kim S, et al. Transposable elements drive widespread expression of oncogenes in human cancers. Nat Genet. 2019;51:611-7.

8. Studer A, Zhao Q, Ross-Ibarra J, Doebley J. Identification of a functional transposon insertion in the maize domestication gene tb1. Nat Genet. 2011;43:1160-3.

9. Salvi S, Sponza G, Morgante M, Tomes D, Niu X, Fengler KA, Meeley R, Ananiev EV, Svitashev S, Bruggemann E, et al. Conserved noncoding genomic sequences associated with a flowering-time quantitative trait locus in maize. Proc Natl Acad Sci U S A. 2007;104:11376-81.

10. Mao H, Wang H, Liu S, Li Z, Yang X, Yan J, Li J, Tran LS, Qin F. A transposable element in a NAC gene is associated with drought tolerance in maize seedlings. Nat Commun. 2015;6:8326.

11. Tian J, Wang C, Xia J, Wu L, Xu G, Wu W, Li D, Qin W, Han X, Chen Q, et al. Teosinte ligule allele narrows plant architecture and enhances high-density maize yields. Science. 2019;365:658-64.

12. Keurentjes JJ, Fu J, Terpstra IR, Garcia JM, van den Ackerveken G, Snoek LB, Peeters AJ, Vreugdenhil D, Koornneef M, Jansen RC. Regulatory network construction in Arabidopsis by using genome-wide gene expression quantitative trait loci. Proc Natl Acad Sci U S A. 2007:104:1708-13.

13. Francesconi $\mathrm{M}$, Lehner $B$. The effects of genetic variation on gene expression dynamics during development. Nature. 2014:505:208-11.

14. Huang W, Carbone MA, Magwire MM, Peiffer JA, Lyman RF, Stone EA, Anholt RR, Mackay TF. Genetic basis of transcriptome diversity in Drosophila melanogaster. Proc Natl Acad Sci U S A. 2015;112:E6010-9.

15. Yu J, Buckler ES. Genetic association mapping and genome organization of maize. Curr Opin Biotechnol. 2006;17:155-60.

16. Liu H, Luo X, Niu L, Xiao Y, Chen L, Liu J, Wang X, Jin M, Li W, Zhang Q, et al. Distant eQTLs and non-coding sequences play critical roles in regulating gene expression and quantitative trait variation in maize. Mol Plant. 2017;10:414-26.

17. Li H, Peng Z, Yang X, Wang W, Fu J, Wang J, Han Y, Chai Y, Guo T, Yang N, et al. Genome-wide association study dissects the genetic architecture of oil biosynthesis in maize kernels. Nat Genet. 2013:45:43-50.

18. Consortium G. Genetic effects on gene expression across human tissues. Nature. 2017;550:204-13.

19. Albert FW, Kruglyak L. The role of regulatory variation in complex traits and disease. Nat Rev Genet. 2015;16:197-212.

20. Gusev A, Ko A, Shi H, Bhatia G, Chung W, Penninx BW, Jansen R, de Geus EJ, Boomsma DI, Wright FA, et al. Integrative approaches for large-scale transcriptome-wide association studies. Nat Genet. 2016;48:245-52.

21. Zhu Z, Zhang F, Hu H, Bakshi A, Robinson MR, Powell JE, Montgomery GW, Goddard ME, Wray NR, Visscher PM, et al. Integration of summary data from GWAS and eQTL studies predicts complex trait gene targets. Nat Genet. 2016;48:481-7.

22. Yang X, Gao S, Xu S, Zhang Z, Prasanna B, Li L, Li J, Yan J. Characterization of a global germplasm collection and its potential utilization for analysis of complex quantitative traits in maize. Mol Breed. 2011;28:511-26.

23. Yang Q, Li Z, Li W, Ku L, Wang C, Ye J, Li K, Yang N, Li Y, Zhong T, et al. CACTA-like transposable element in ZmCCT attenuated photoperiod sensitivity and accelerated the postdomestication spread of maize. Proc Natl Acad Sci U S A. 2013:110:16969-74.

24. Wen W, Li D, Li X, Gao Y, Li W, Li H, Liu J, Liu H, Chen W, Luo J, et al. Metabolome-based genome-wide association study of maize kernel leads to novel biochemical insights. Nat Commun. 2014;5:3438.

25. Jiao Y, Peluso P, Shi J, Liang T, Stitzer MC, Wang B, Campbell MS, Stein JC, Wei X, Chin CS, et al. Improved maize reference genome with single-molecule technologies. Nature. 2017;546:524-7.

26. Chiang HH, Hwang I, Goodman HM. Isolation of the Arabidopsis GA4 locus. Plant Cell. 1995;7:195-201.

27. Tanaka K, Asami T, Yoshida S, Nakamura Y, Matsuo T, Okamoto S. Brassinosteroid homeostasis in Arabidopsis is ensured by feedback expressions of multiple genes involved in its metabolism. Plant Physiol. 2005;138:1117-25.

28. Su H, Cao Y, Ku L, Yao W, Ren Z, Dou D, Wang H, Liu H, Tian L, Zheng Y, et al. Dual functions of ZmNF-YA3 in photoperiod-dependent flowering and abiotic stress responses in maize. J Exp Bot. 2018;69:5177-89.

29. Larson ER, Van Zelm E, Roux C, Marion-Poll A, Blatt MR. Clathrin heavy chain subunits coordinate endo- and exocytic traffic and affect Stomatal movement. Plant Physiol. 2017;175:708-20

30. Liu D, Gong Q, Ma Y, Li P, Li J, Yang S, Yuan L, Yu Y, Pan D, Xu F, et al. cpSecA, a thylakoid protein translocase subunit, is essential for photosynthetic development in Arabidopsis. J Exp Bot. 2010;61:1655-69.

31. Moneo-Sanchez M, Alonso-Chico A, Knox JP, Dopico B, Labrador E, Martin I. B-(1,4)-Galactan remodelling in Arabidopsis cell walls affects the xyloglucan structure during elongation. Planta. 2019;249:351-62.

32. Matiolli CC, Tomaz JP, Duarte GT, Prado FM, Del Bem LE, Silveira AB, Gauer L, Correa LG, Drumond RD, Viana AJ, et al. The Arabidopsis bZIP gene AtbZIP63 is a sensitive integrator of transient abscisic acid and glucose signals. Plant Physiol. 2011;157:692-705.

33. Yang Y, Qin Y, Xie C, Zhao F, Zhao J, Liu D, Chen S, Fuglsang AT, Palmgren MG, Schumaker KS, et al. The Arabidopsis chaperone J3 regulates the plasma membrane $\mathrm{H}^{+}$-ATPase through interaction with the PKS5 kinase. Plant Cell. 2010;22: 1313-32. 
34. Zhu JK. Abiotic stress signaling and responses in plants. Cell. 2016;167:313-24.

35. Song L, Huang SC, Wise A, Castanon R, Nery JR, Chen H, Watanabe M, Thomas J, Bar-Joseph Z, Ecker JR. A transcription factor hierarchy defines an environmental stress response network. Science. 2016;354:aag1550.

36. Chen H, Wang JP, Liu H, Li H, Lin YJ, Shi R, Yang C, Gao J, Zhou C, Li Q, et al. Hierarchical transcription factor and chromatin binding network for wood formation in black cottonwood (Populus trichocarpa). Plant Cell. 2019;31:602-26.

37. Peng Z, Serino G, Deng XW. Molecular characterization of subunit 6 of the COP9 signalosome and its role in multifaceted developmental processes in Arabidopsis. Plant Cell. 2001;13:2393-407.

38. Yoshida T, Fujita Y, Maruyama K, Mogami J, Todaka D, Shinozaki K, Yamaguchi-Shinozaki K. Four Arabidopsis AREB/ABF transcription factors function predominantly in gene expression downstream of SnRK2 kinases in abscisic acid signalling in response to osmotic stress. Plant Cell Environ. 2015;38:35-49.

39. Gondolf VM, Stoppel R, Ebert B, Rautengarten C, Liwanag AJ, Loque D, Scheller HV. A gene stacking approach leads to engineered plants with highly increased galactan levels in Arabidopsis. BMC Plant Biol. 2014;14:344.

40. Nziengui H, Bouhidel K, Pillon D, Der C, Marty F, Schoefs B. Reticulon-like proteins in Arabidopsis thaliana: structural organization and ER localization. FEBS Lett. 2007;581:3356-62.

41. Okamoto M, Tanaka Y, Abrams SR, Kamiya Y, Seki M, Nambara E. High humidity induces abscisic acid 8'-hydroxylase in stomata and vasculature to regulate local and systemic abscisic acid responses in Arabidopsis. Plant Physiol. 2009;149: 825-34.

42. Gao MJ, Lydiate DJ, Li X, Lui H, Gjetvaj B, Hegedus DD, Rozwadowski K. Repression of seed maturation genes by a trihelix transcriptional repressor in Arabidopsis seedlings. Plant Cell. 2009;21:54-71.

43. Lee HK, Cho SK, Son O, Xu Z, Hwang I, Kim WT. Drought stress-induced Rma1H1, a RING membrane-anchor E3 ubiquitin ligase homolog, regulates aquaporin levels via ubiquitination in transgenic Arabidopsis plants. Plant Cell. 2009;21:622-41.

44. Chen HY, Huh JH, Yu YC, Ho LH, Chen LQ, Tholl D, Frommer WB, Guo WJ. The Arabidopsis vacuolar sugar transporter SWEET2 limits carbon sequestration from roots and restricts Pythium infection. Plant J. 2015;83:1046-58.

45. Yamaguchi-Shinozaki K, Shinozaki K. Transcriptional regulatory networks in cellular responses and tolerance to dehydration and cold stresses. Annu Rev Plant Biol. 2006;57:781-803.

46. Sun S, Zhou Y, Chen J, Shi J, Zhao H, Song W, Zhang M, Cui Y, Dong X, Liu H, et al. Extensive intraspecific gene order and gene structural variations between Mo17 and other maize genomes. Nat Genet. 2018;50:1289-95.

47. Yang N, Liu J, Gao Q, Gui S, Chen L, Yang L, Huang J, Deng T, Luo J, He L, et al. Genome assembly of a tropical maize inbred line provides insights into structural variation and crop improvement. Nat Genet. 2019;51:1052-9.

48. Su W, Gu X, Peterson T. TIR-learner, a new ensemble method for TIR transposable element annotation, provides evidence for abundant new transposable elements in the maize genome. Mol Plant. 2019;12:447-60.

49. Peng Y, Xiong D, Zhao L, Ouyang W, Wang S, Sun J, Zhang Q, Guan P, Xie L, Li W, et al. Chromatin interaction maps reveal genetic regulation for quantitative traits in maize. Nat Commun. 2019;10:2632.

50. Dong T, Park Y, Hwang I. Abscisic acid: biosynthesis, inactivation, homoeostasis and signalling. Essays Biochem. 2015;58:29-48.

51. Thatcher SR, Zhou W, Leonard A, Wang BB, Beatty M, Zastrow-Hayes G, Zhao X, Baumgarten A, Li B. Genome-wide analysis of alternative splicing in Zea mays: landscape and genetic regulation. Plant Cell. 2014;26:3472-87.

52. Pertea M, Kim D, Pertea GM, Leek JT, Salzberg SL. Transcript-level expression analysis of RNA-seq experiments with HISAT, StringTie and Ballgown. Nat Protoc. 2016;11:1650-67.

53. Kim D, Langmead B, Salzberg SL. HISAT: a fast spliced aligner with low memory requirements. Nat Methods. 2015;12 357-60.

54. Liao Y, Smyth GK, Shi W. featureCounts: an efficient general purpose program for assigning sequence reads to genomic features. Bioinformatics. 2014;30:923-30.

55. Robinson MD, McCarthy DJ, Smyth GK. edgeR: a bioconductor package for differential expression analysis of digital gene expression data. Bioinformatics. 2010;26:139-40.

56. Pertea M, Pertea GM, Antonescu CM, Chang TC, Mendell JT, Salzberg SL. StringTie enables improved reconstruction of a transcriptome from RNA-seq reads. Nat Biotechnol. 2015;33:290-5.

57. McKenna A, Hanna M, Banks E, Sivachenko A, Cibulskis K, Kernytsky A, Garimella K, Altshuler D, Gabriel S, Daly M, et al. The genome analysis toolkit: a MapReduce framework for analyzing next-generation DNA sequencing data. Genome Res. 2010;20:1297-303.

58. Li H, Handsaker B, Wysoker A, Fennell T, Ruan J, Homer N, Marth G, Abecasis G, Durbin R. The sequence alignment/map format and SAMtools. Bioinformatics. 2009;25:2078-9.

59. Zhang Z, Ersoz E, Lai CQ, Todhunter RJ, Tiwari HK, Gore MA, Bradbury PJ, Yu J, Arnett DK, Ordovas JM, et al. Mixed linear model approach adapted for genome-wide association studies. Nat Genet. 2010;42:355-60.

60. Raj A, Stephens M, Pritchard JK. fastSTRUCTURE: variational inference of population structure in large SNP data sets. Genetics. 2014;197:573-89.

61. Stegle O, Parts L, Piipari M, Winn J, Durbin R. Using probabilistic estimation of expression residuals (PEER) to obtain increased power and interpretability of gene expression analyses. Nat Protoc. 2012;7:500-7.

62. Bradbury PJ, Zhang Z, Kroon DE, Casstevens TM, Ramdoss Y, Buckler ES. TASSEL: software for association mapping of complex traits in diverse samples. Bioinformatics. 2007;23:2633-5.

63. Barrett JC, Fry B, Maller J, Daly MJ. Haploview: analysis and visualization of LD and haplotype maps. Bioinformatics. 2005; 21:263-5.

64. Xing HL, Dong L, Wang ZP, Zhang HY, Han CY, Liu B, Wang XC, Chen QJ. A CRISPR/Cas9 toolkit for multiplex genome editing in plants. BMC Plant Biol. 2014;14:327.

65. Wang Y, Song F, Zhu J, Zhang S, Yang Y, Chen T, Tang B, Dong L, Ding N, Zhang Q, et al. GSA: genome sequence archive." Genomics Proteomics Bioinformatics. 2017;15:14-8.

66. Song S, Tian D, Li C, Tang B, Dong L, Xiao J, Bao Y, Zhao W, He H, Zhang Z. Genome variation map: a data repository of genome variations in BIG Data Center. Nucleic Acids Res. 2018;46:D944-D49.

67. Members BDC. Database resources of the BIG Data Center in 2019. Nucleic Acids Res. 2019;47:D8-D14.

68. Liu S, Li C, Wang H, Wang S, Yang S, Liu X, Yan J, Li B, Beatty M, Zastrow-Hayes G, Song S, Qin F. Mapping regulatory variants controlling gene expression in drought response and tolerance in maize. RNA-sequencing data, BIGD: GSA: CRA000334. https://bigd.big.ac.cn/gsa/browse/CRA000334 (2020a). Accessed 9 Aug 2017. 
69. Liu S, Li C, Wang H, Wang S, Yang S, Liu X, Yan J, Li B, Beatty M, Zastrow-Hayes G, Song S, Qin F. Mapping regulatory variants controlling gene expression in drought response and tolerance in maize. RNA-sequencing reads and gene expression level (FPKM) in each sample, BIGD: GSA: CRA002002. https://bigd.big.ac.cn/gsa/browse/CRA002002 (2020b). Accessed 24 Sept 2019

70. Liu S, Li C, Wang H, Wang S, Yang S, Liu X, Yan J, Li B, Beatty M, Zastrow-Hayes G, Song S, Qin F. Mapping regulatory variants controlling gene expression in drought response and tolerance in maize. Genotypic data, BIGD: gvm: GVM000048. https://bigd.big.ac.cn/gvm/getProjectDetail?project=GVM000048 (2020c). Accessed 27 Sept 2019.

71. Liu S, Li C, Wang H, Wang S, Yang S, Liu X, Yan J, Li B, Beatty M, Zastrow-Hayes G, Song S, Qin F. Mapping regulatory variants controlling gene expression in drought response and tolerance in maize. The script for the MR analysis, Github: https://github.com/whweve/MPGV (2020d). Accessed 10 March 2020.

72. Liu S, Li C, Wang H, Wang S, Yang S, Liu X, Yan J, Li B, Beatty M, Zastrow-Hayes G, Song S, Qin F. Mapping regulatory variants controlling gene expression in drought response and tolerance in maize. The RNA-seq reads, NCBI: BioProject: PRJNA637522. https://www.ncbi.nlm.nih.gov/bioproject/PRJNA637522 (2020e). Accessed 1 Jun 2020.

\section{Publisher's Note}

Springer Nature remains neutral with regard to jurisdictional claims in published maps and institutional affiliations.

Ready to submit your research? Choose BMC and benefit from:

- fast, convenient online submission

- thorough peer review by experienced researchers in your field

- rapid publication on acceptance

- support for research data, including large and complex data types

- gold Open Access which fosters wider collaboration and increased citations

- maximum visibility for your research: over $100 \mathrm{M}$ website views per year

At $\mathrm{BMC}$, research is always in progress.

Learn more biomedcentral.com/submissions 